\title{
Applicability of the ultra-high radio frequency band (UHF) for hydrometeor detection by phase difference method
}

\author{
Peter FABO ${ }^{1}$ (D), Pavol NEJEDLÍK ${ }^{2, *}$ (D), Michal KUBA ${ }^{1}$ (D), \\ Milan ONDERKA ${ }^{2}$ (D), Dušan PODHORSKÝ ${ }^{2}$ (D) \\ ${ }^{1}$ University of Žilina, Slovak Republic \\ ${ }^{2}$ Earth Science Institute of the Slovak Academy of Sciences, \\ Dúbravská cesta 9, Bratislava, Slovak Republic
}

\begin{abstract}
Hydrometeors (rain, fog and ice crystals) affect the transmission of electromagnetic signals. Previous research showed that alterations in the signal (amplitude and phase) are affected by the composition of the atmosphere, e.g. the presence of hydrometeors. The majority of hydrometeorological detecting methods are based on the attenuation of electromagnetic signals as they penetrate the atmosphere. Novel methods based on monitoring of parameters of the signal appeared in recent time. This article presents the first results from our investigation of how hydrometeors affect the phase differences in signals transmitted by BTS stations. Cell phone operators transmit electromagnetic signals in the $1 \mathrm{GHz}$ frequency band. This paper describes a novel concept of how phase differences between two signals arriving at two different antennas can be used to detect hydrometeors. Although the described concept is assumed to be independent from the signal strength, the analysed signal must be detectable. The primary advantage of the proposed passive method is that the signal is almost ubiquitous and does not require demodulation. In densely populated areas, the network of BTS stations reaches a spatial density of 1 station per $1 \mathrm{~km}^{2}$ which gives excellent opportunity to use the signal for detection purposes.
\end{abstract}

Key words: electromagnetic signal, refraction, phase, hydrometeors, BTS

\section{Introduction}

Detection of hydrometeors (rain, ice crystals, fog) in the atmosphere is based on two different physical principles: (1) mechanical capture of falling raindrops and snowflakes, and (2) measuring changes in the emitted electromagnetic or optical signals. The first conventional method is used in the network of ground-based rain gauges, whereas the second method is used in

\footnotetext{
*corresponding author: e-mail: nejedlik@yahoo.com
} 
e.g. meteorological radars, remote sensing, optical methods, i.e., measuring the attenuation or changes of electromagnetic or optical signals penetrating the atmosphere. Real-time ground-based observations of atmospheric precipitation are usually carried out in a network of pluviometers (or rain gauges) equipped with on-line data transfer. Such pluviometers represent point-measurements of precipitation. However, fluctuation in the intensity of precipitation and the wind field around the pluviometer cause the systematic errors that can reach up to 10\% (Sevruk, 1996). On the other hand, meteorological radars monitor the whole space within the radius of more than $100 \mathrm{~km}$, yet they have their own limitations as well, e.g. ground obstacles and the blind zone below a certain height above the ground where the detection is impossible (Collier, 1989). The height of the blind zone depends on the character of the terrain and can reach up to about 300-700 m. In contrast to this disadvantage of meteorological radars, radio waves can be detected by ground-based communication transmitters placed at heights of a few tenths of meters in order to cover the blind zone.

Detection of rainfall in the boundary layer of the atmosphere is based on the evaluation of electromagnetic smog coming out of communication facilities such as mobile phones, WIFI etc. It can render important information that could not be obtained by any of the above mentioned methods. Making use of the electromagnetic smog can therefore contribute to a better detection of precipitation. It is a well-known fact that an electromagnetic signal is attenuated as it travels through a medium (atmosphere). However, the relation between attenuation/signal drop-out and precipitation intensity appears to be linear in contrast to the highly non-linear relation between rainfall intensity and reflected signal of meteorological radars (Leijnse et al., 2007). That is why the first methods applied for the detection of hydrometeors in the atmosphere were based on the attenuation of electromagnetic signals between a transmitter and a receiver. The applicability of the attenuation of a radio signal for estimating rainfall intensity received attention of several investigators in the 1960s (Hogg, 1968; Semplak and Turrin, 1969). Several authors focused on the range of frequencies between 15 and $35 \mathrm{GHz}$. Some pioneering works from the 1970s and 1980s pointed out on the existence of a quasi linear relationship between rainfall intensity and the attenuation of the electromagnetic field. But as noted by Rahimi et al. (2003), although this relationship is not always linear, as their experimen- 
tal research showed, it is plausible to achieve better detection of rainfall when parallel measurements using two frequencies are conducted. Additional information from the measurements of intensive rainfall appear to be important at the analysis of intensive precipitation with small-sized drops (Brauer et al., 2011) and also in dense populated areas with complicated infrastructure (Overeem et al., 2011). Nevertheless, this technique requires a rather demanding knowledge about the source of signal which is not always available. It is also limited to a certain frequency range. As shown in Fig. 1, the signal attenuation is very low at frequencies below $3 \mathrm{GHz}$, hence it is not possible to use these frequencies to detect hydrometeors using the attenuation method (Fagiani et al., 2018). Nevertheless, it is possible to utilize the time delay of the signal caused by changes in refraction.

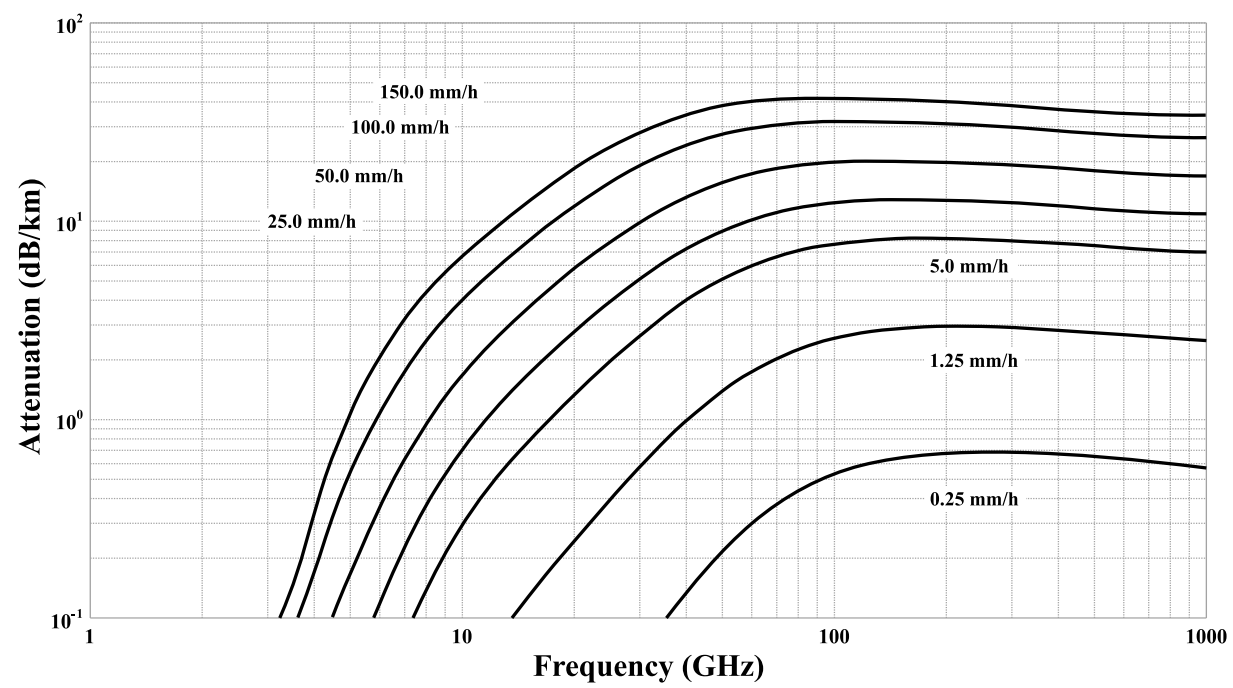

Fig. 1. Signal attenuation caused by rainfall. Source: Fagiani et al. (2018).

Watson and Coleman (Watson and Coleman, 2010) suggest to use the $\mathrm{DAB}$ and/or DVB-T signal to estimate the water vapour content in the atmosphere by measuring atmospheric refractivity. They described the process of continual monitoring of the phase of carrier frequency by extracting the pilot symbols from DAB (Digital Audio Broadcasting) signal. This algorithm requires signal demodulation in order to reconstruct the in-built synchronization (pilot) symbols. The phase changes are estimated by the phase-locked loop (PLL) or by some another phase estimation technique. 
Kawamura et al. (2017) found that the propagation delay of digital terrestrial broadcasting waves can be related to the content of water vapour in the respective volume of the atmosphere. They measured the time delay of the reflected signal between the receiver and the reflector in the form of the phase delay which is obtained as a phase in the maximum of the side lobe magnitude of the complex time-lag (power delay) profile. Time-lag profiles are derived by the inverse Fourier transform of the OFDM (Orthogonal Frequency Division Multiplexing) synchronization symbols in the received ISBD-T signals. ISBD-T is a digital television terrestrial broadcasting standard used in Japan. It follows that the algorithm also needs some demodulation of the signal. The phase changes caused by the signal delay changes between the receiver and the reflector are measured in the millimetres order.

Recently, Kuba et al. (2020) reviewed the applicability of cellular network signals for atmospheric phenomena detection. In this paper the method of phase differences is explored in terms of its applicability for hydrometeor detection.

\section{Theoretical background of the phase differences method}

The principle of the method is based on measuring differences in the signal arriving at two different points (antennas) along two different trajectories. In contrary to the above mentioned methods, no time standard is required here. This assumption is based on the expectation that the existing technology is able to select the fitted trajectory of the signal due to the adequate parameters of the antennas. The signals arriving at each antenna are evaluated by a coherent signal receiver. Such processing produces the time difference attributed to the period of the signal as a phase difference which is proportional to the difference of the effective refractivity of each trajectory. In contrast to the previous methods when absolute parameters of the atmosphere are used, this method describes the relative changes between the source of a signal and the receiver within the respective space up to 100 $\mathrm{m}$ above the ground.

According to several authors (e.g., Grabner and Kvicera, 2011), straightline propagation of an electromagnetic signal through the atmosphere is modified by refraction. Refraction is caused by variations in air density and 
is a function of altitude. Generally, refraction is also a function of temperature and its gradient, air pressure, and the amount of liquid and solid phases of water in the atmosphere. The refractive index of the air without hydrometeors $n=1.000320$. For the sake of practicality, the refractive index $N=(n-1) \cdot 10^{6}$ is usually applied. It has to be noted here that the refraction depends also on signal frequency. Refraction of air without hydrometeors at frequencies below $100 \mathrm{GHz}$ can be calculated using the formula proposed by Bean and Dutton (1966):

$N=\frac{77.6}{T} \cdot\left(P+4810 \frac{e}{T}\right)-4.03 \cdot 10^{7} \frac{N_{e}}{f^{2}}$,

where $T[\mathrm{~K}]$ is air temperature, $P[\mathrm{hPa}]$ is air pressure, $e[\mathrm{hPa}]$ is the partial pressure of water vapour, $N_{e}$ is the number of density of free electrons per $\mathrm{m}^{3}$ and $f$ is frequency of the signal in $\mathrm{Hz}$. Vertical changes of these atmospheric parameters define the variability in refraction. Refraction of $-40 \mathrm{~N} / \mathrm{km}$ is taken as a standard value, however, it depends on the local conditions. Refraction gradient determines the trajectory of the electromagnetic signal in the atmosphere, as illustrated in Fig. 2.

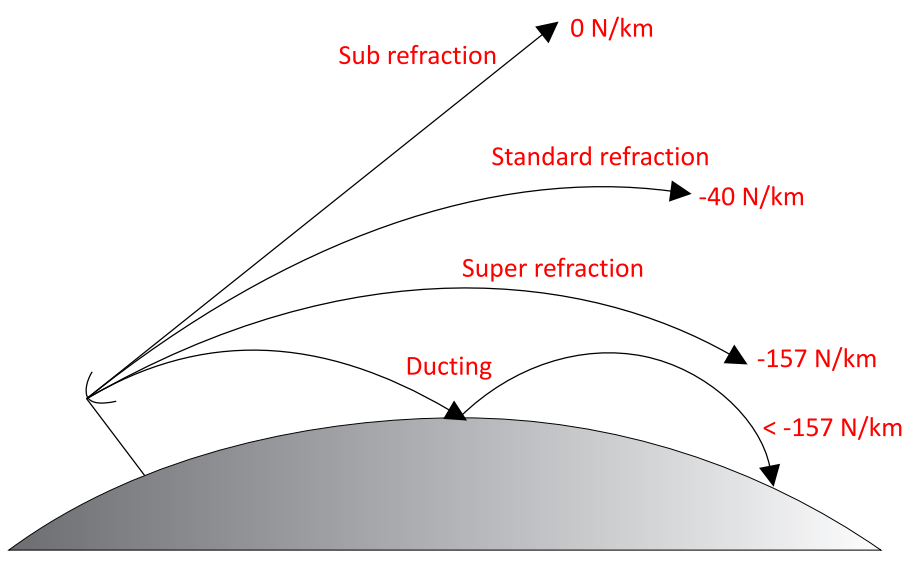

Fig. 2. Trajectories of electromagnetic signal in the atmosphere.

To describe the propagation of an electromagnetic signal in the atmosphere the so-called modified refraction $M$ is applied. $M$ considers the Earth's curvature. To account for the Earth's curvature effect, the modified refraction $M$ is defined as: 
$M=N+\left(\frac{z}{R e}\right) 10^{6}$,

where $z[\mathrm{~m}]$ is altitude and $R e[\mathrm{~m}]$ is the Earth's radius.

There is no clear consensus as to how to calculate the profile of refraction in an atmosphere containing hydrometeors. Different simulation models based on experimental observations estimate the vertical profile of refraction over the sea level. For example, the simple univariate model of Paulus and Jeske (Paulus, 1990) considers different effects of the atmospheric parameters on the modified refraction within the defined layers:

$M_{(z)}=M_{(0)}+0.125\left(z-z_{(d)}\right) \ln \left(\frac{z+0.00015}{0.00015}\right)$,

where $z_{(d)}$ represents an elevation below which the refraction is predominantly influenced by concentration of water vapour due to evaporation, while the effect of air pressure dominates at higher altitudes.

The least-time principle suggests that an electromagnetic signal travels between two points along a line $y=y(x)$ with the shortest time. The Euler-Lagrange equation applied to the function $f\left(x, y, y^{\prime}\right)$ to account for the space properties is defined, in the case of two dimensional space, as:

$$
\frac{\mathrm{d}}{\mathrm{d} x}\left(\frac{\partial f}{\partial y^{\prime}}\right)=\frac{\partial}{\partial y} \text {. }
$$

For the special case of a constant vertical gradient independent from the $x$-axis an analytical solution is given by the assumption of a vertical refraction profile $n=n(y)=n_{0}+\alpha y$ where $\alpha$ is the refractivity gradient and $y$ is the height above the ground. It is assumed that in this configuration a fictive beam of electromagnetic energy is released from point $\mathrm{A}$ at $\zeta_{0}$ angle (Fig. 3).

The beam trajectory between the points $\mathrm{A}$ and $\mathrm{B}$ can be described as:

$f\left(x, y, y^{\prime}\right)=\frac{n(y)}{c} \sqrt{1+y^{\prime}}$.

The solution does not depend on the $x$-axis value. In this case the EulerLagrange equation is set to:

$f-y^{\prime} \frac{\partial f}{\partial y^{\prime}}=\frac{1}{k}$, 


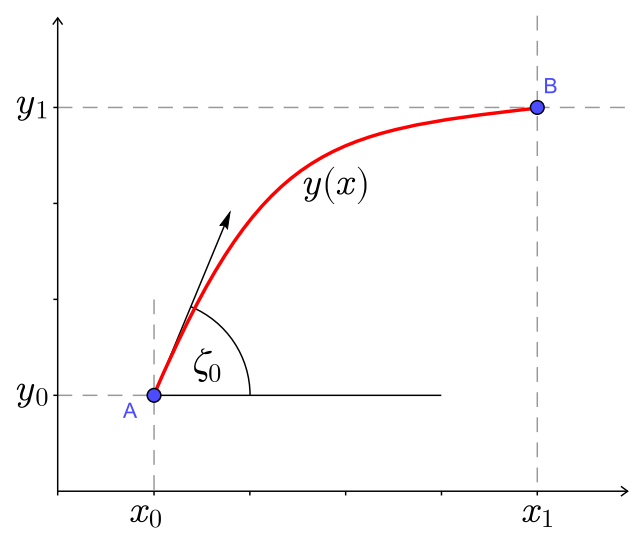

Fig. 3. Beam trajectory.

where $k$ is an integral constant the value of which can be obtained from the approximation of the space by creating fictive horizontal layers with constant refraction (Fig. 4). The integral constant is then incorporated by the following formula:

$n(y) \cos (\zeta)=n_{0} \cos \left(\zeta_{0}\right)=\frac{c}{k}$,

$\zeta_{0}$ represents the angle of the beam in point A. By substituting we get the Euler-Lagrange equation in the following form:

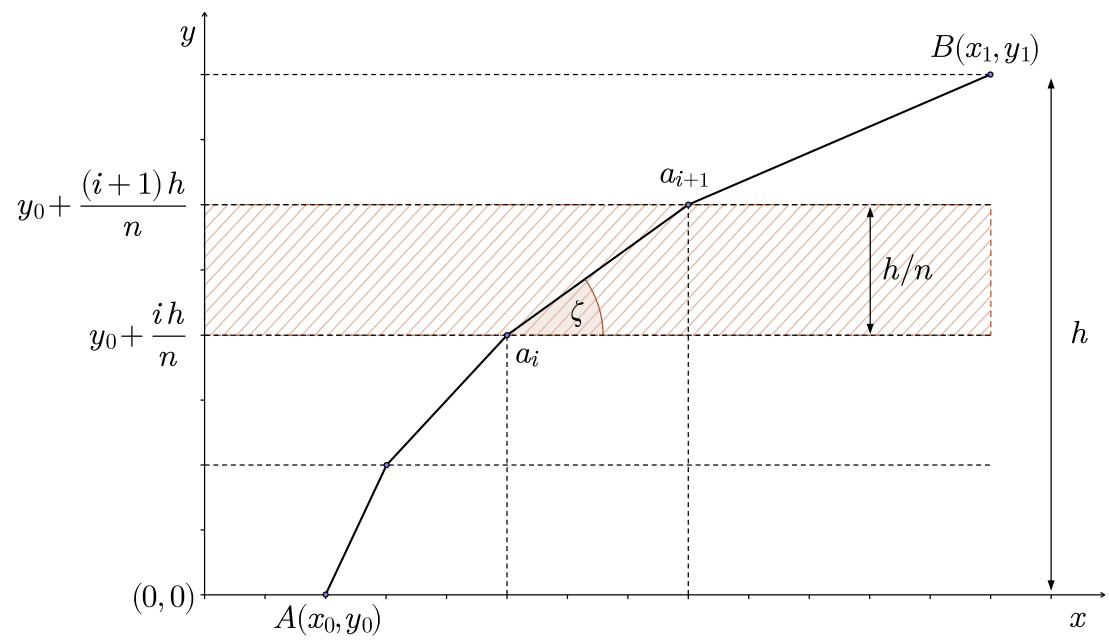

Fig. 4. Beam trajectory in layers. 
$y=-\frac{n_{0}}{\alpha}+\frac{n_{0} \cos \left(\zeta_{0}\right)}{\alpha} \cosh \left(\frac{\alpha x}{n_{0} \cos \left(\zeta_{0}\right)}+\zeta_{0}\right)$.

The previously listed theory describes the beam trajectory only in a trivial case. Nevertheless, it can be used to estimate the trajectory and to interpret some partial results of the experiments. In order to define the character of the trajectory, optical $S$ and geometric $L$ length of a trajectory are defined as:

$$
\begin{aligned}
& S= \int_{s} \mathrm{~d} s=\int_{x_{0}}^{x_{1}} \sqrt{1+\left(y^{\prime}\right)^{2}} \mathrm{~d} x=\int_{x_{0}}^{x_{1}} \sqrt{1+\sinh ^{2}\left(\frac{\alpha x}{n_{0} \cos \left(\zeta_{0}\right)}+\zeta_{0}\right)} \mathrm{d} x, \\
& L= \int_{s} n(s) \mathrm{d} s=\int_{x_{0}}^{x_{1}}\left(n_{0}+\alpha y\right) \sqrt{1+\left(y^{\prime}\right)^{2}} \mathrm{~d} x= \\
&= \frac{n_{0}}{\alpha} \int_{x_{0}}^{x_{1}}\left(-1+\cos \left(\zeta_{0}\right) \cosh \left(\frac{\alpha x}{n_{0} \cos \left(\zeta_{0}\right)}+\zeta_{0}\right)\right) \times \\
& \times \sqrt{1+\sinh ^{2}\left(\frac{\alpha x}{n_{0} \cos \left(\zeta_{0}\right)}+\zeta_{0}\right)} \mathrm{d} x .
\end{aligned}
$$

The optical path length is equivalent to the trajectory which would be taken in the space with constant refractive index. The ratio $L / S$ defines the effective refraction of respective trajectory.

Let us assume that the source of the signal is placed at points $\mathrm{A}$ and $\mathrm{C}$ and two antennas are located at points $\mathrm{B}$ and $\mathrm{D}$. Once $n_{a}$ and $n_{c}$ are the effective refractive indices along the respective trajectories:

$n_{a}=\frac{L_{A B}}{S_{A B}}, \quad n_{c}=\frac{L_{C D}}{S_{C D}}$,

the times difference in the signal arriving to the points $\mathrm{B}$ and $\mathrm{C}$ will be as follows:

$\Delta t=\frac{L_{A B}-L_{A C}}{c}=\frac{n_{a} S_{A B}-n_{c} S_{C D}}{c}=\frac{n_{a} s_{a}+q \lambda+\Delta s-n_{c} s_{c}-k \lambda}{c}$,

where the length $n_{a} S_{A B}$ is expressed as a sum of multiples of wave-length $\lambda$ and the offset $n_{a} s_{a}$. The distance between antennas is $\Delta s$ and $q, k$ are integers. Considering the difficulties associated with a direct measurement of time shifts in the UHF band we can convert this problem to estimating the mutual phase differences detected by a coherent receiver. A phase difference can be obtained after signal processing: 


$$
\begin{aligned}
\Delta \phi & =2 \pi \frac{\Delta t}{T_{c}}=2 \pi \frac{\left[n_{a} s_{a}+q \lambda+\Delta s-n_{c} s_{c}-k \lambda\right]}{c T_{c}}= \\
& =2 \pi(q+k)+2 \pi \frac{\Delta s+\Delta x\left(n_{a}, n_{c}\right)}{\lambda}=\phi_{s}+\Delta \phi_{n} .
\end{aligned}
$$

The Paulus-Jeske model can be used to solve this problem. The gradient of refractive index calculated for layers close to the ground was used to obtain the shape of simulated trajectories (Fig. 5):

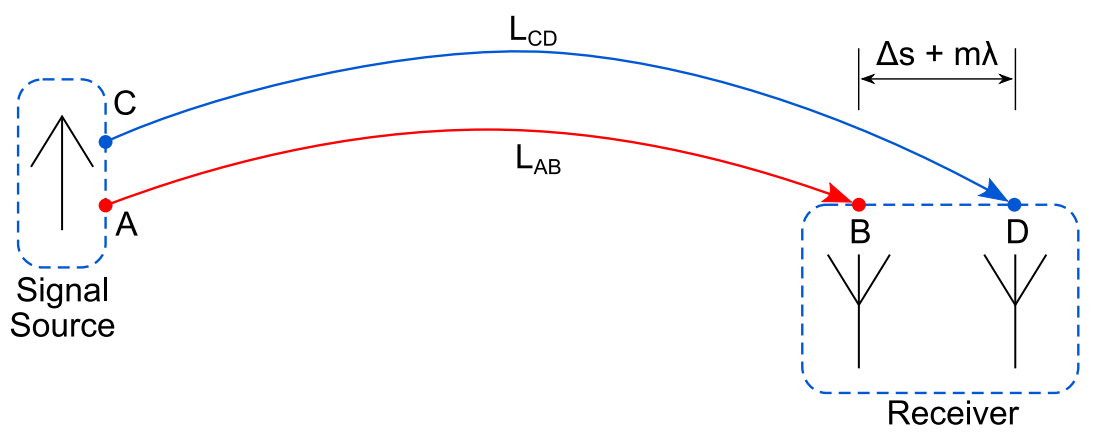

Fig. 5. Trajectories and phase difference.

The outputs from simulations carried out for a 1000 metre distance of the signal source and $\Delta s=5 \mathrm{~m}$ distance between the antennas give 0.065 rad.

\section{Experimental verification of the theory}

Data from the Jaslovské Bohunice meteorological station operated by the Slovak Hydrometeorological Institute were used to verify the presented phase difference method. A meteorological mast with the height of 200 metres is located at the ground-based meteorological station. Sensors measuring air temperature, humidity, wind direction and wind velocity are placed at multiple levels so that it was possible to retrieve the vertical profile of the respective atmospheric parameters. A transmitter operating at $935 \mathrm{MHz}$ was placed on the mast at a height of 30 metres above the ground. The antennas were placed at a roof of the administrative building at a distance of $150 \mathrm{~m}$ from the mast (Fig. 6).

The experimental setup comprises a two-antenna array, a two-channel coherent receiver and a computer for the data processing and evaluation. 

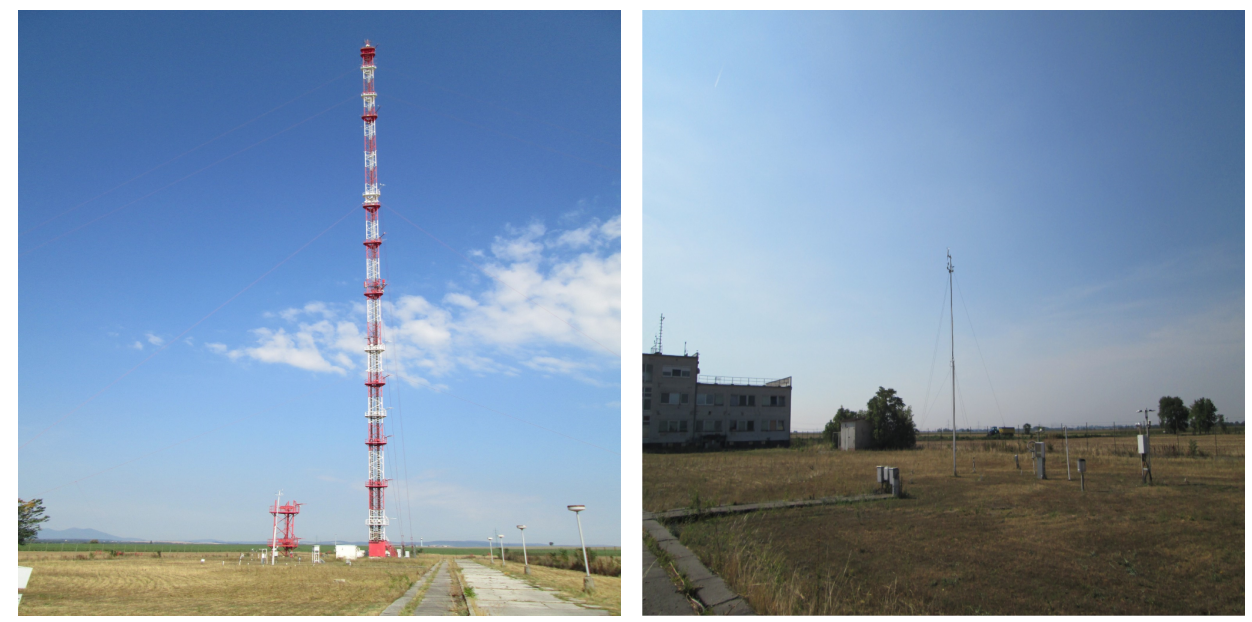

Fig. 6. Meteorological mast, ground meteorological station and the administrative building of Jaslovské Bohunice station, Slovakia.

The following block diagram (Fig. 7) shows the simplified arrangement of the measurement system:

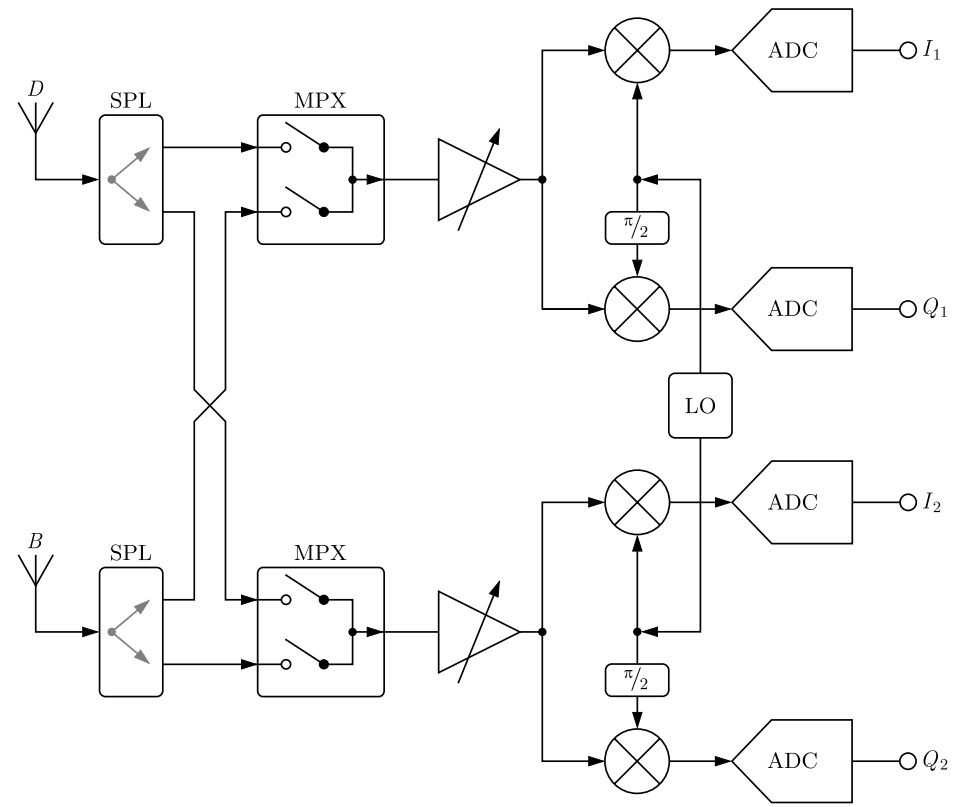

Fig. 7. Measuring device scheme. 
The signals received by the antennas continue to input circuits consisting of an antenna splitter (SPL) and a multiplexer (MPX). The signals then go into two identical SDR receivers (i.e., two-channel coherent SDR receiver) where the quadrature multiplier units have a common local oscillator (LO). This enables the coherent processing of the signals. The digitized signal in the form of $\mathrm{I}-\mathrm{Q}$ components from each channel is evaluated by a computer. The same computer simultaneously handles the measurement configuration by switching the channels by the MPX. In this way it is possible to compensate the unwanted internal phase shifts caused by the differences in the frequency response of both SDR channels and different lengths of the antenna cables. Figure 8 shows a spectral density distribution of both channels towards the carrier frequency. The spectrum of the I-Q signals at the output of each channel of the coherent SDR receiver contains all signal sources surrounding the antennas in the whole frequency range of the receiver.

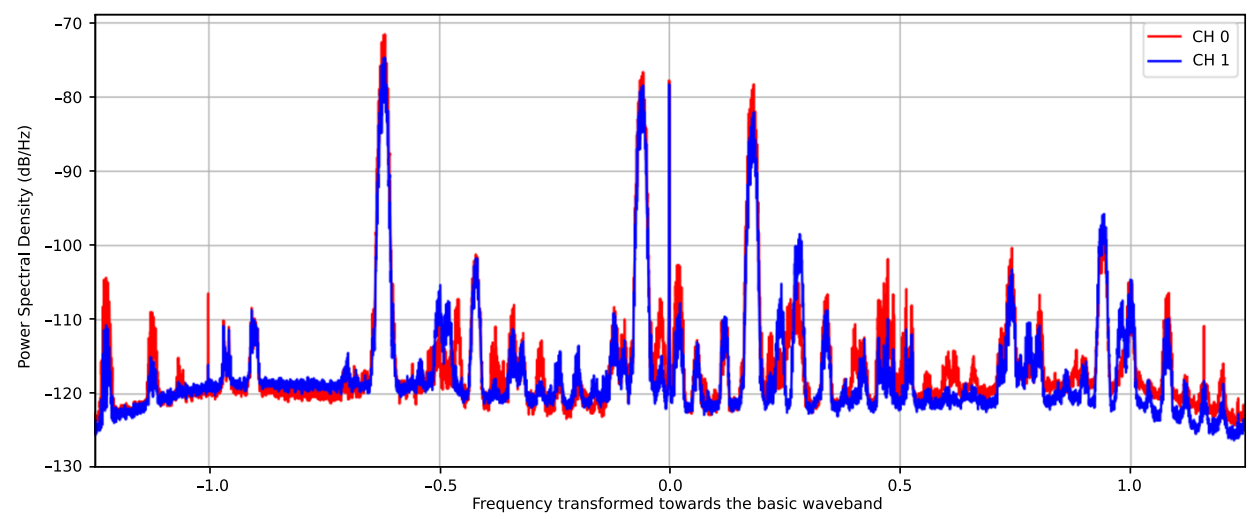

Fig. 8. Spectral density distribution.

The final evaluation of the signals by a computer requires the spectrum frequency shift of the desired signal to the baseband followed by lowpass filtering to eliminate the unwanted parts of the spectra. After filtration and the signal downsampling the final calculation of phase difference between channels is performed according to the following relation:

$\Delta \phi=\operatorname{Arg}\left\{\sum_{n=0}^{N-1} x[n] y^{*}[n]\right\}$,

where $n$ is the discrete time index, $N$ is the length of the block of complex 
I-Q signal samples $x[n]$ and $y[n]$ from both channels of the coherent SDR receiver, $(\cdot)^{*}$ is the complex-conjugate operator and $\operatorname{Arg}\{\cdot\}$ is the operator which returns the argument (phase) of the complex number.

To sum up, the proposed method has the following properties:

- It is a fully passive method - no self-radiation - it utilizes radiation from other sources;

- No data streams decoding required - no legislative restrictions;

- The method creates virtual sensors among signal sources and receiving antennas;

- Simultaneous measurements in multiple directions are possible;

- Independence on modulation formats, type of service, etc.;

- Real time detection.

The processing of signals from SDR receiver in real-time is a demanding task. In the frequency band of 920-940 MHz (i.e., $20 \mathrm{MHz}$ bandwidth the downlink part of GSM G2 band) the data rate from SDR receiver to the computer reaches $400 \mathrm{Mbyte} / \mathrm{sec}$. We decided that every second only one data block of 20 millisecond duration is processed by this experimental setup.

Figures 9-10 show the diurnal evolution of the vertical profile of air temperature and humidity, the estimated refractivity at $30 \mathrm{~m}$ above the ground and the phase difference for March 24, 2020. The weather conditions during the experiment were characterized with clear skies, long duration of sunshine and no rainfall. The synoptic situation was characterized by a large-scale anticyclone with its centre located over the Baltic region. The dominant airflow was blowing from the north-east bearing cold and relatively dry air.
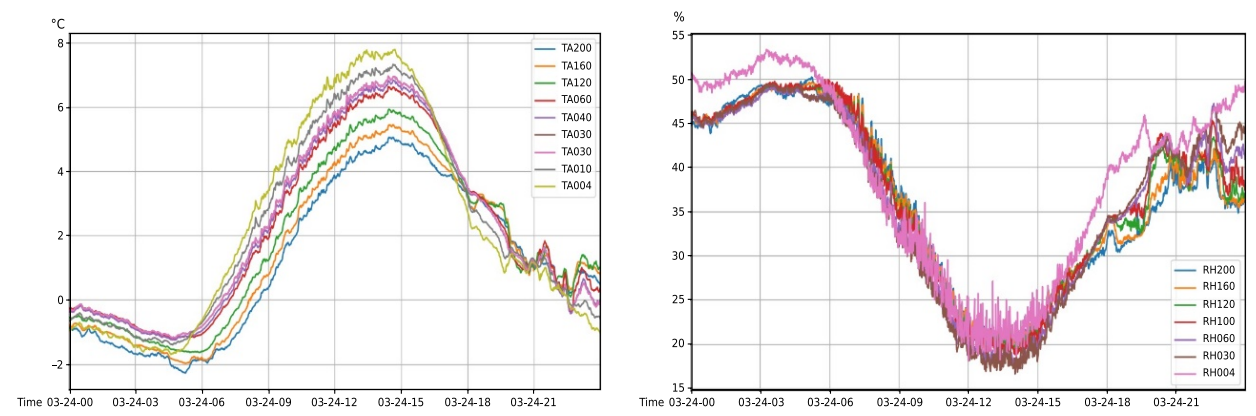

Fig. 9. Temperature and relative humidity profiles on March 24, 2020. 


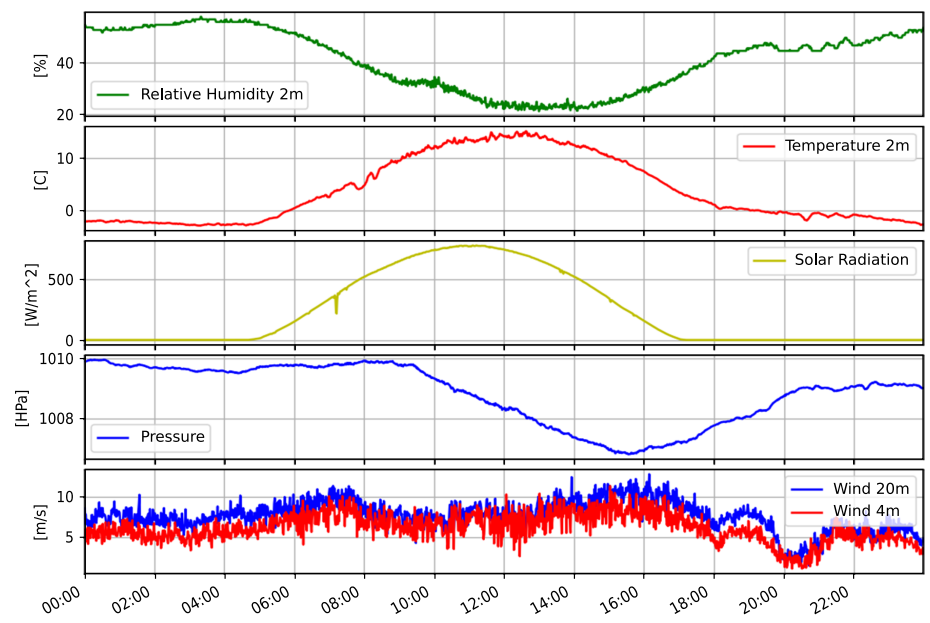

Fig. 10. Diurnal evolution of selected meteorological parameters (March 24, 2020). The numbers after $\mathrm{TA}$ and $\mathrm{RH}$ in upper figures indicate the position of the temperature and relative humidity sensors above the ground.

The diurnal evolution of air temperature was typical for days with nighttime and early morning inversions. There was a well-developed vertical air temperature gradient with a temperature maximum in early afternoon. A sufficient income of solar energy heated the already relatively dry air and the relative humidity dropped to less than $25 \%$ around midnight. The temperature gradient near the ground and the wind speed of approx. $10 \mathrm{~m} / \mathrm{s}$ caused a slight turbulence and water vapour content fluctuation in this layer.

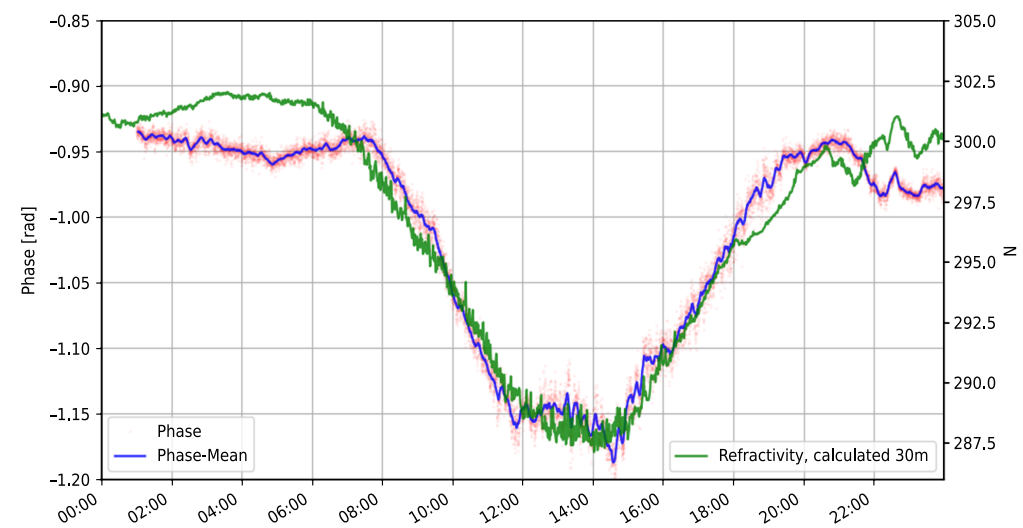

Fig. 11. The comparison of refractivity and phase (March 24, 2020). 
The phase shift basically corresponds to the refractivity value of air without hydrometeors, as shown in Fig. 11. The fluctuations after 12 a.m. correspond to the fluctuations of water vapour.

\section{Response of the signal to rain}

Disturbance of electromagnetic signals in the atmosphere is caused mostly by water in liquid and solid state. Rain and snow significantly change the refractive index and affect the signal trajectory. Figures 12 and 13 provide
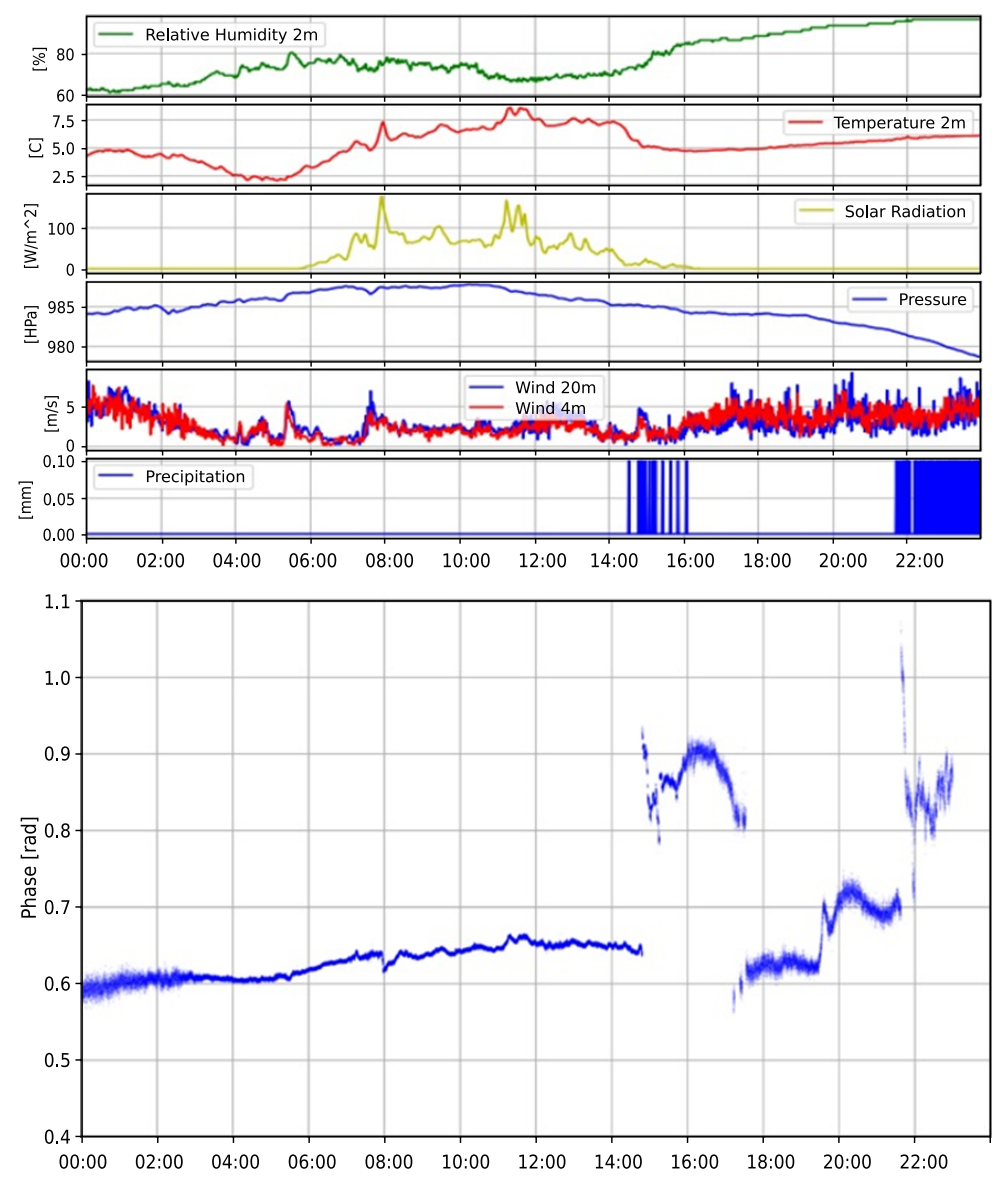

Fig. 12. Evolution of selected meteorological parameters and the signal response on March $1,2020$. 


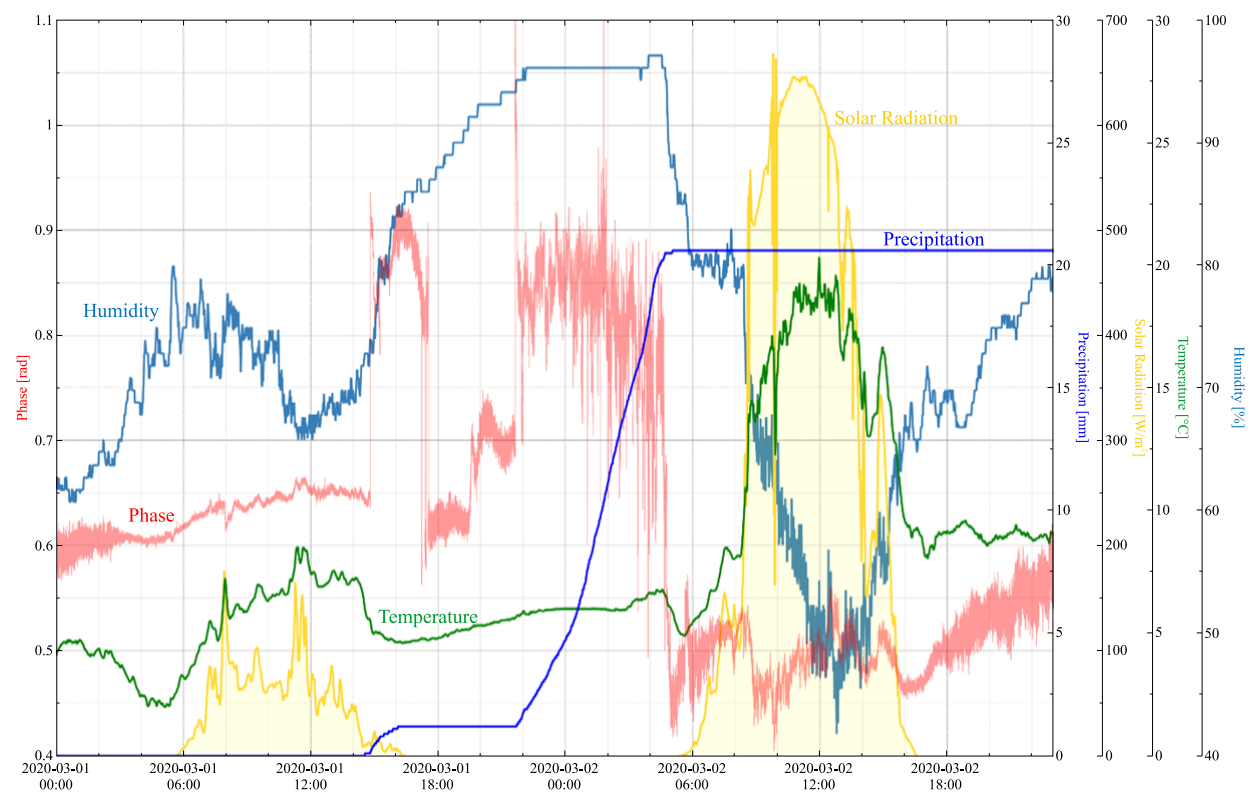

Fig. 13. Cumulative graph of the course of meteorological parameters and the signal response on March 1-2, 2020.

an example of such signal alteration during the passage of a rain arising from the cold front on March 1, 2020. Meteorological data were taken from the nearby ground station at Jaslovské Bohunice, the transmitter and receiver antennas were located at the same place as during the experiment of March 24, 2020. The synoptic situation was characterized by an extensive anticyclone with its centre located over Baltic countries. The sky was fully overcast, the intrusion of cold air was week and the partial water vapour pressure and the relative humidity were steadily increasing. A low pressure zone remained over the territory for the next two days but the air pressure changes were insignificant. Sharp response of the signal (phase) is clearly detectable at the start and the end of the rain episode.

Further variations during the rain period respond to the rain intensity. Phase variations out of the rain periods are much less developed. This gives the opportunity to detect the liquid water in the air and also to monitor the changing rain intensity. However, intensive insolation after the sunrise on March 2 (see Fig. 13), which started shortly after the end of rainfall, and declining insulation in the late afternoon caused strong variations in 
relative humidity. This causes much smaller phase variations during this period than the rain occurrence.

The previously described process shows the potential of the phase difference method to monitor the occurrence of liquid water in the selected volume of the atmosphere along the path line of the sight between the signal source and the receiver. Although the results interpret the precipitation occurrence only in a qualitative form, the results do express a certain quantitative view regarding the rain intensity fluctuations.

\section{Discussion and conclusions}

In this paper, we focused on how the physical state of the atmosphere affects the phase differences between two electromagnetic signals propagated by different trajectories in a heterogenous environment of the atmosphere. Our findings suggest that different natural and technical parameters affect the shape of the signal trajectories. Hence their direct assessment from just the phase difference is problematic and biased with uncertainties. Nevertheless, it is clear that phase difference provides information on how the refractivity evolves in time reflecting the instantaneous physical conditions of the atmosphere. The phase difference is affected by both the physical state of the atmosphere and by the processes occurring in it. Based on the conducted experiments the basic characteristics of phase differences method can be formulated as follows:

- the phase difference method is a passive method using external sources of electromagnetic signal;

- this method enables to monitor the state of the atmosphere (presence of hydrometeors) based on the differences in signals propagated along two different trajectories;

- phase difference between the signals is related to the frequency of the source signal and depends on the properties of the atmosphere;

- phase difference consists of constant $\phi_{s}$ and variable $\phi_{n}$ components;

- the constant component $\phi_{s}$ includes a phase shift given by the geometry of the system configuration. It includes also all technical aspects related to the signal transmission such as the different length of antenna cable, delay in the input circuit, their adjustment etc.;

- the variable component $\phi_{n}$ depends on the difference of effective refrac- 
tions of the trajectories (i.e., local gradient of the refraction); on the projection of the distance of the antennas to the direction towards the signal source; on the absolute distance antennas from the signal source.

It is obvious that the measured values correspond to the integral amount of precipitation in between the transmitter and the receiver (antennas). This gives us one dimensional information about the occurrence and intensity of the precipitation. By setting at least three couples of antennas in a selected space and/or by utilizing the signal from multiplied BTS stations it will be possible to get an areal view of the precipitation field.

Various methods to detect atmospheric properties can be applied by using the characteristics of an electromagnetic signal penetrating a certain part of the atmosphere. The proposed concept of phase differences gives the opportunity to develop a fully passive detection method with no need to decode the signal. No knowledge of the signal source parameters is needed. However, in order to correctly interpret the phase difference, one needs an adequate receiver that can detect the phase difference between the signals received at a minimum of two antennas. Depending on the signal receiver the described method of phase differences makes it possible to monitor physical parameters within a thin layer of only a few tenths of meters over the ground. One of the advantages of this method is that it does not require any additional technical information on the signal source. The method relies only on the signal availability in the band around $1 \mathrm{MHz}$ operated by cell phone operators. Since cell phone service providers operate almost everywhere in densely populated areas, the state of the atmosphere and the presence of hydrometeors can be monitored in real time.

Acknowledgements. The research presented in this paper was supported by following grants: VEGA grant 2/0015/18 "Meso- and micro-meteorological exploration of the occurrence of hydrometeors in boundary layer of troposphere based on passive evaluation of changes of electromagnetic radiation from anthropogenic sources", VEGA grant No. 2/0003/21 "Complex analysis of the effects of rising air temperature on rainfall extremes in Slovakia", R\&D Incentives contract "Expanding the base of theoretical hypotheses and initiating assumptions to ensure scientific progress in methods of monitoring hydrometeors in the lower troposphere", Operational Programme Integrated Infrastructure project: "Research and development of contactless methods for obtaining geospatial data for forest monitoring to improve forest management and enhance forest protection", ITMS code 313011V465 (The project is co-funded by European Regional Development Fund). Authors also thank to Slovak Hydrometeorological Institute and their workers in 
Jaslovské Bohunice and Gánovce and Astronomical Institute of SAS at Skalnaté pleso and Stará Lesná for data and technical support during the measuring campaigns.

\section{References}

Bean B. R., Dutton E. J., 1966: Radio Meteorology. National Bureau of Standards Monograph 92. Library of Congress Catalog Card No. 65-60033.

Brauer C. C., Teuling A. J., Overeem A., van der Velde Y., Hazenberg P., Warmerdam P. M. M., Uijlenhoet R., 2011: Anatomy of extraordinary rainfall and flash flood in a Dutch lowland catchment. Hydrol. Earth Syst. Sci., 15, 1991-2005, doi: $10.5194 /$ hess-15-1991-2011.

Collier C. G., 1989: Applications of Weather Radar Systems. Ellis Horwood, Chichester, England, $294 \mathrm{p}$.

Fagiani A., Vogel M., Arismar Cerqueira S., 2018: Material Characterization and Propagation Analysis of mm-Waves Indoor Networks. J. Microw. Optoelectron. Electromagn. Appl., 17, 4, São Caetano do Sul, Oct./Dec. 2018, 628-637, doi: 10.1590/21 79-10742018v17i41548.

Grabner M., Kvicera V., 2011: Atmospheric refraction and propagation in lower troposphere. In: Zhurbenko V. (Ed.): Electromagnetic Waves, IntechOpen, 139-156, doi: 10.5772/16379, available from: https://www.intechopen.com/books/electr omagnetic-waves/atmospheric-refraction-and-propagation-in-lower-troposp here.

Hogg D. C., 1968: Millimeter-wave communication through the atmosphere. Science, 159, 3810, 39-46, doi: 10.1126/science.159.3810.39.

Kawamura S., Ohta H., Hanado H., Yamamoto M. K., Shiga N., Kido K., Yasuda S., Goto T., Ichikawa R.,. Amagai J., Imamura K., Fujieda M., Iwai H., Sugitani S., Iguchi T., 2017: Water vapor estimation using digital terrestrial broadcasting waves. Radio Science, 52, 3, 367-377, doi : 10.1002/2016RS006191.

Kuba M., Fabo P., Podhorský D., Faktorová D., Savin A., 2020: Passive method for monitoring atmospheric phenomena by evaluating the cellular network signals. In: Tian G. Y., Gao B. (Eds.): Electromagnetic non-destructive evaluation (XXIII), 1st edition, Amsterdam, IOS Press, ISBN: 978-1-64368-118-4, 269-276, doi: 10.3233/saem 200044.

Leijnse H., Uijlenhoet R., Stricker J. N. M., 2007: Rainfall measurement using radio links from cellular communication networks. Water Resour. Res., 43, 3, W03201, doi: 10.1029/2006WR005631.

Overeem A., Leijnse H., Uijlenhoet R., 2011: Measuring urban rainfall using microwave links from commercial cellular communication networks. Water Resour. Res., 47, 12, W12505, doi: 10.1029/2010WR010350.

Paulus R. A., 1990: Evaporation duct effects on sea clutter. IEEE Trans. Antennas Propag., 38, 11, 1765-1771, doi: 10.1109/8.102737.

Rahimi A. R., Holt A. R., Upton G. J. G., Cummings R. J., 2003: Use of dual-frequency microwave links for measuring path-averaged rainfall. J. Geophys. Res., 108, D15, 
4467, doi: 10.1029/2002JD003202.

Semplak R. A., Turrin R. H., 1969: Some measurements of attenuation by rainfall at 18.5 GHz. Bell Syst. Tech. J., 48, 6, 1767-1787, doi: 10.1002/j.1538-7305.1969. tb011 51.x.

Sevruk B., 1996: Adjustment of tipping-bucket precipitation gauge measurements. Atmos. Res., 42, 1-4, 237-246, doi : 10.1016/0169-8095(95)00066-6.

Watson R. J., Coleman C. J., 2010: The use of signals of opportunity for the measurement of atmospheric refractivity. Proceedings of the 4th European Conference on Antennas and Propagation, CCIB, Barcelona, Spain, 12-16 April 2010, Publisher: IEEE, ISBN: 978-84-7653-472-4. 